\title{
A comparative study on the use of mannitol and dexamethasone in severe perinatal asphyxia among newborn babies conducted in Ad-din Sakina Medical College, Jessore
}

\author{
KZ Hossain ${ }^{1 凶}$, MA Islam², PK Das ${ }^{3}$
}

\begin{abstract}
We conducted a comparative study on sixty full-term newborn babies with cerebral edema and severe perinatal asphyxia treating with mannitol or dexamethasone in Ad-din Sakina Medical College Hospital, Jessore. The age (in hours) of the children in both groups was similar. The score for clinical condition of the children with perinatal asphyxia at 24 hours, 48 hours and 72 hours after using mannitol were significantly lower than the respective score at 24 hours, 48 hours and 72 hours after using dexamethasone ( $p<0.01, p<0.05$ and $p<0.001$, respectively). The score for bulging condition of anterior fontanelle of the children with perinatal asphyxia at 24 hours, 48 hours and 72 hours after using mannitol were significantly lower than the respective score at 24 hours, 48 hours and 72 hours after using dexamethasone $(p<0.05, p<0.05$ and $p<$ 0.001 , respectively). The score for clinical condition of the children with perinatal asphyxia at 24 hours, 48 hours and 72 hours after using mannitol were significantly lower than the respective score before the treatment $(p<0.05, p<0.001$ and $p<0.001$, respectively). The scores at 48 hours and 72 hours after using dexamethasone were significantly lower than the respective score before the treatment $(p<0.001$ and $p<0.001$, respectively). The score for bulging condition of anterior fontanelle of the children with perinatal asphyxia at 24 hours, 48 hours and 72 hours after using mannitol were significantly lower than the respective score before the treatment $(p<$ $0.001, p<0.001$ and $p<0.001$, respectively). The score at 48 hours and 72 hours after using dexamethasone were significantly lower than the respective score before the treatment $(p<$ 0.001 and $p<0.001$, respectively). Mannitol seems to be more beneficial than dexamethasone in the management of severe perinatal asphyxia due to raised intracranial pressure associated with cerebral edema.
\end{abstract}

Keywords: severe perinatal asphyxia, mannitol, dexamethasone, comparison.

\section{Introduction}

Perinatal asphyxia is a common problem of neonates. Severe perinatal asphyxia is a major cause of morbidity and mortality in the neonatal period. Perinatal asphyxia remains a concerned issue in health circles of the developing countries.
The incidence of birth asphyxia causing a cerebral disturbance is unknown but is probably in the order of 1.5 to 6 per 1000 live births. It is arguably the commonest cause of perinatal brain injury associated with a longterm neurological handicap. ${ }^{1}$ The management of severe birth asphyxia is both

1. KZ Hossain, MBBS, DCH, Assistant Professor of Pediatrics, Ad-din Sakina Medical College, Jessore.

Email: sstationary9@gmail.com

2. MA Islam, MBBS, DCH, Assistant Professor of Pediatrics, Gazi Medical College, Khulna

3. PK Das, MBBs, Medical Officer, Department of Pediatrics, Ad-din Sakina Medical College, Jessore 
supportive and directed towards avoidance of, or treatment for, cerebral edema. Supportive treatment includes control of convulsions, avoidance of hypotension, and prevention of metabolic disturbances. Where intracranial hypertension is thought to be present, dehydration, corticosteroids, and osmotic agents have been recommended. ${ }^{2}$ It was reported that dexamethasone and mannitol have an impact on lowering raised intracranial pressure as measured directly from the subarachnoid space. ${ }^{3}$

Many term newborns suffer some degree of perinatal asphyxia, but few become permanently brain damaged as a consequence. The newborns at risk for major neurologic handicaps have evidence of derangement in many organs, depressed cerebral function at birth that continues for days or weeks, and in many cases, convulsions soon after birth. The pathophysiologic mechanism of brain damage in hypoxic-ischemic encephalopathy remains contested, and no specific therapy is clearly beneficial. ${ }^{4}$ The immediate treatment of the asphyxiated term newborn requires attention to the derangements in multiple organ systems and the use of anticonvulsants when indicated. ${ }^{5-7}$ The delay involved in obtaining informed parental consent for such trials might obscure a clinically important therapeutic effect. ${ }^{8}$

Both dexamethasone and mannitol cause a reduction in intracranial pressure with a simultaneous reduction in systemic blood pressure. ${ }^{2}$ In the case of mannitol, this blood pressure reduction seems to be transient with subsequent recovery and improvement in cerebral perfusion pressure, but there is no improvement in cerebral perfusion pressure over six hours after dexamethasone treatment and subsequent improvement may be due to the use of mannitol. ${ }^{2}$ Intravenous use of $20.0 \%$ mannitol reduces morbidity and mortality in severe perinatal asphyxia. $^{3,9}$

We conducted a comparative study on sixty full-term severely asphyxiated newborn babies with cerebral edema to compare the usefulness of mannitol or dexamethasone in severe perinatal asphyxia.

\section{Materials and Method}

A comparative study on sixty full-term newborn babies with cerebral edema and severe and severe perinatal asphyxia treating with mannitol or dexamethasone was conducted in Ad-din Sakina Medical College Hospital, Jessore during the period from July 2016 to December 2016.

For comparison, 30 patients were selected randomly to treatment with mannitol and 30 patients were selected randomly to treat with dexamethasone. Severely asphyxiated neonates were given $20.0 \%$ intravenous mannitol $2 \mathrm{gm} / \mathrm{kg} /$ day for 3 days. ${ }^{9}$ The other 30 neonates were given dexamethasone, and regularly clinical progress of both groups of neonates was followed up for 3 days. Scores for clinical condition (presence of three symptoms together $=5$, two symptoms $=4$, single symptom $=2$ and no symptom $=0$ ) and bulging condition of anterior fontanelle (highly bulged $=3$, slightly bulged $=2$, tensed $=1$ and normal $=0$ ) of the children with perinatal asphyxia at before treatment, 24 hours, 48 hours and 72 hours after treatment using mannitol or dexamethasone.

Data were collected, compiled and entered in a spreadsheet (Microsoft Excel) and analyzed using Statistical Package for Social Sciences (SPSS), version 16. ${ }^{10}$ The comparison between the two treatment conditions by mannitol and dexamethasone using data at before treatment, and 24 hours, 48 hours and 72 hours points after treatment was done by Student's t-test, and the comparison within the two treatment conditions by mannitol or dexamethasone using data at before treatment compared with 24 hours, 48 hours and 72 hours points after treatment was done by one way analysis of variance with Bonferroni correction. Results were reported mean \pm standard error (SE). The level of $p$ value < 0.05 was considered significant.

\section{Results}

Table 1 shows the age of the children (in hours). There was no significant difference in age of the children of the groups $(p>0.05)$.

Table 2 shows the scores for clinical condition of the children with perinatal asphyxia before and after treatment using mannitol and dexamethasone. The score at 24 hours, 48 hours and 72 hours after using mannitol were significantly lower than the respective score at 24 hours, 48 hours and 72 hours after using dexamethasone $(p<0.01, p<$ 0.05 and $p<0.001$, respectively). 
Table 1. Age of the children (in hours)

\begin{tabular}{lrrr}
\hline Group & Number & $\begin{array}{r}\text { Age in hours } \\
(\text { mean } \pm \text { SE) }\end{array}$ & p value \\
\hline Mannitol & 30 & $22.4 \pm 3.5$ & $>0.05$ \\
Dexamethasone & 30 & $20.5 \pm 3.4$ & \\
\hline
\end{tabular}

SE, standard error. Statistical analysis by Student's $t$-test.

Table 2. Scores for clinical condition of the children with perinatal asphyxia before and after treatment using mannitol and dexamethasone

\begin{tabular}{lrrrr}
\hline & Number & $\begin{array}{r}\text { Mannitol } \\
(\text { mean } \pm S E)\end{array}$ & $\begin{array}{r}\text { Dexamethasone } \\
(\text { mean } \pm S E)\end{array}$ & $p$ value \\
\hline Before treatment & 30 & $4.5 \pm 0.1$ & $4.6 \pm 0.1$ & $>0.05$ \\
24 hours after treatment & 30 & $4.1 \pm 0.1$ & $4.5 \pm 0.1$ & $<0.01$ \\
48 hours after treatment & 30 & $2.7 \pm 0.2$ & $3.2 \pm 0.2$ & $<0.05$ \\
72 hours after treatment & 30 & $0.0 \pm 0.0$ & $1.8 \pm 0.2$ & $<0.001$ \\
\hline
\end{tabular}

SE, standard error. Statistical analysis by Student's $t$-test.

Table 3. Scores for bulging of anterior frontanelle of the children with perinatal asphyxia before and after treatment using mannitol and dexamethasone

\begin{tabular}{lrrrr}
\hline & Number & $\begin{array}{r}\text { Mannitol } \\
(\text { mean } \pm \text { SE) }\end{array}$ & $\begin{array}{r}\text { Dexamethasone } \\
(\text { mean } \pm S E)\end{array}$ & $p$ value \\
\hline Before treatment & 30 & $2.1 \pm 0.1$ & $2.3 \pm 0.1$ & $>0.05$ \\
24 hours after treatment & 30 & $1.3 \pm 0.1$ & $2.0 \pm 0.1$ & $>0.05$ \\
48 hours after treatment & 30 & $0.8 \pm 0.1$ & $1.3 \pm 0.1$ & $>0.05$ \\
72 hours after treatment & 30 & $0.0 \pm 0.0$ & $0.6 \pm 0.1$ & $<0.001$ \\
\hline
\end{tabular}

SE, standard error. Statistical analysis by Student's $t$-test.

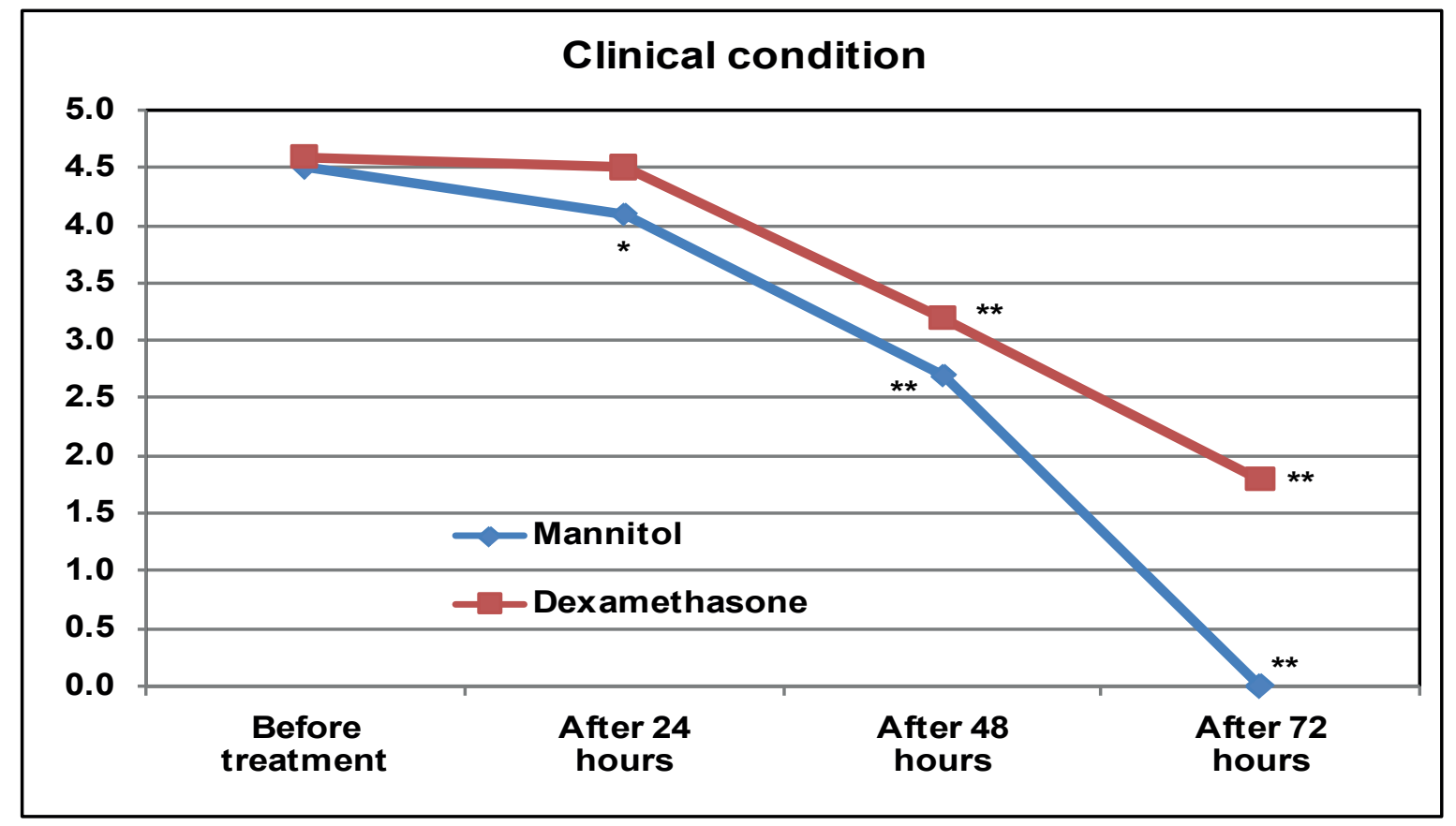

Fig. 1. Clinical condition of the children with perinatal asphyxia before and after treatment using mannitol and dexamethasone. One way analysis of variance revealed

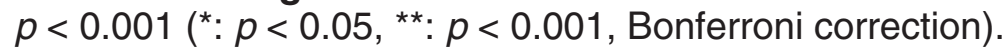




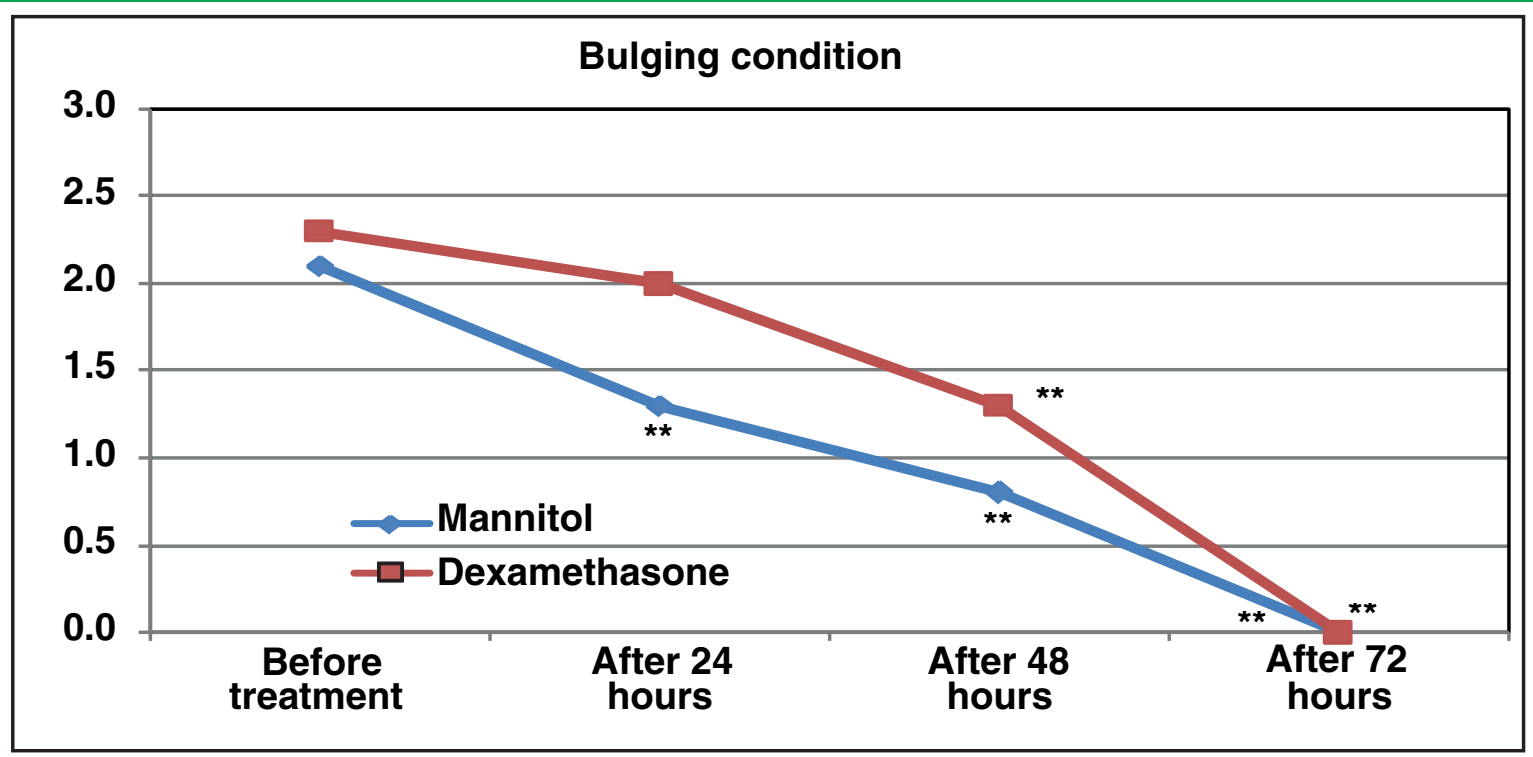

Fig. 2. Bulging condition of the children with perinatal asphyxia before and after treatment using mannitol and dexamethasone. One way analysis of variance revealed $p<0.001{ }^{* *}: p<0.001$, Bonferroni correction).

Table 3 shows the scores for bulging condition of anterior fontanelle of the children with perinatal asphyxia before and after treatment using mannitol and dexamethasone. The score at 24 hours, 48 hours and 72 hours after using mannitol were significantly lower than the respective score at 24 hours, 48 hours and 72 hours after using dexamethasone $(p<0.05, p<0.05$ and $p<0.001$, respectively).

Fig. 1 shows the clinical condition of the children with perinatal asphyxia before and after treatment using mannitol and dexamethasone. The score at 24 hours, 48 hours and 72 hours after using mannitol were significantly lower than the respective score before the treatment $(p<0.05, p<0.001$ and $p<0.001$, respectively). The score at 48 hours and 72 hours after using dexamethasone were significantly lower than the respective score before the treatment $(p<$ 0.001 and $p<0.001$, respectively).

Fig. 2 shows the bulging condition of anterior fontanelle of the children with perinatal asphyxia before and after treatment using mannitol or dexamethasone. The score at 24 hours, 48 hours and 72 hours after using mannitol were significantly lower than the respective score before the treatment $(p<$ $0.001, p<0.001$ and $p<0.001$, respectively). The score at 48 hours and 72 hours after using dexamethasone were significantly lower than the respective score before the treatment $(p<0.001$ and $p<0.001$, respectively).

\section{Discussion}

Perinatal asphyxia used to refer to a critical lack of oxygen to the fetus during labor and delivery, which is ischemic encephalopathy and refers to the clinical disturbance of the brain functions that may follow hypoxic ischemia, moderate and severe grades of perinatal asphyxia. Mannitol infusion is effective in lowering intracranial pressure. Mannitol is a white crystalline solid that looks like fine sugar, tastes sweet like sucrose metabolized in the liver, and excretes through the kidney. Half-life is 100 minutes. Usually used to reduce acutely raised intracranial pressure, certain cases of kidney failure with low urine output, decrease pressure in the eye, increase elimination of certain toxins, also acts as an osmotic laxative when used orally. Serious side effects are worsening heart failure, electrolytes abnormalities or low blood volume. It is contraindicated in patients with anuria, congestive heart failure, and active cerebral hemorrhage.

We conducted a comparative study on sixty full-term newborn babies with cerebral edema and severe perinatal asphyxia treating with mannitol or dexamethasone in 
Ad-din Sakina Medical College Hospital, Jessore. The age (in hours) of the children in both groups was similar.

The score for clinical condition of the children with perinatal asphyxia at 24 hours, 48 hours and 72 hours after using mannitol were significantly lower than the respective score at 24 hours, 48 hours and 72 hours after using dexamethasone. The score for bulging condition of anterior fontanelle of the children with perinatal asphyxia at 24 hours, 48 hours and 72 hours after using mannitol were significantly lower than the respective score at 24 hours, 48 hours and 72 hours after using dexamethasone. The score for clinical condition of the children with perinatal asphyxia at 24 hours, 48 hours and 72 hours after using mannitol were significantly lower than the respective score before the treatment. The scores at 48 hours and 72 hours after using dexamethasone were significantly lower than the respective score before the treatment.

The score for bulging condition of anterior fontanelle of the children with perinatal asphyxia at 24 hours, 48 hours and 72 hours after using mannitol were significantly lower than the respective score before the treatment. The score at 48 hours and 72 hours after using dexamethasone were significantly lower than the respective score before the treatment.

After 3 days of given intravenous mannitol the patients rapidly regained consciousness and convulsion was controlled in those neonates who were given intravenous mannitol, but the other group of neonates who were given dexamethasone improved very slowly or become static in some cases during the observation period.

In a study, it has been reported that mannitol therapy did not ameliorate the incidence, distribution, or severity of tissue injury in the cerebral cortex, subcortical white matter, hippocampus, striatum, or thalamus of the ipsilateral cerebral hemisphere compared with the controls. Mannitol substantially reduces the extent of cerebral edema follow- ing hypoxia-ischemia. ${ }^{11}$ We can find little to support the routine use of dexamethasone in severe perinatal asphyxia but mannitol infusion seems to be more beneficial than dexamethasone in the management of severe perinatal asphyxia due to raised intracranial pressure associated with cerebral edema.

\section{Conclusion}

Use of mannitol infusion in severe perinatal asphyxia seems to be more effective and beneficial than dexamethasone in lowering intracranial pressure and improving cerebral perfusion pressure by decreasing cerebral edema.

\section{References}

1. Volpe JJ. Neurology of the newborn. Philadeiphia: WB Saunders, 1981.

2. Levene MI, Evans DH. Medical management of raised intracranial pressure after severe birth asphyxia. Arch Dis Child 1985;60(1):12-6.

3. Levene MI, Evans DH. Continuous measurement of subarachnoid pressure in the severely asphyxiated newborn. Arch Dis Child 1983;58:1013-5.

4. Fenichel GM. Hypoxic-ischemic encephalopathy in the newborn. Arch Neurol 1983;40(5):261-6.

5. Vannucci RC. Current and potentially new management strategies for perinatal hypoxicischemic encephalopathy. Pediatrics 1990;85(6):961-8.

6. Whitelaw A, Thoresen M. Clinical trials of treatments after perinatal asphyxia. Curr Opin Pediatr 2003;14(6):664-8.

7. Whitelaw A. Systematic review of therapy after hypoxic-ischemic brain injury in the perinatal period. Semin Neonatol 2000;5(1):33-40.

8. Vannucci RC, Perlman JM. Interventions for perinatal hypoxic-ischemic encephalopathy. Pedatrics 1997;100(6):1004-14.

9. Tavakkoli $F$. Review of the role of mannitol in the therapy of children. 18th Expert Committee on the Selection and Use of Essential Medicines. Section 16: Diuretics- Mannitol review (Children), pp. 1-43, 2011.

10.Statistical Package for Social Sciences (SPSS) version 16 for Windows. Chicago: SPSS; 2008.

11.Mujsce DJ, Towfighi J, Stern D, Vannucci RC. Mannitol therapy in perinatal hypoxic-ischemic brain damage in rats. Stroke 1990;21(8):1210-4.

\section{Suggestion for citation of the above:}

Hossain KZ, Islam MA, Das PK. A comparative study on the use of mannitol and dexamethasone in severe perinatal asphyxia among newborn babies conducted in Ad-din Sakina Medical College, Jessore. Mediscope 2018;5(2):16-20. 